\title{
La nanotecnología y sus posibilidades de aplicación en el campo científico-tecnológico
}

\author{
Nanotechnology and its possibilities of application in the \\ scientific-technological field
}

\author{
Yoerquis Mejias Sánchez'; Niviola Cabrera Cruz"'; Ana Margarita Toledo \\ Fernández"'; Orgel J osé Duany Machado'v \\ 'Especialista de I Grado en Pediatría. Instructor. Dirección Nacional de Ciencia y \\ Técnica. MINSAP. La Habana, Cuba. \\ "Especialista de II Grado en Epidemiología. Dirección Nacional de Ciencia y Técnica. \\ MINSAP. La Habana, Cuba. \\ IIIEspecialista de II Grado en Medicina General Integral. Investigadora Agregada. \\ Dirección Nacional de Ciencia y Técnica. MINSAP. La Habana, Cuba. \\ IVEspecialista de II Grado en Epidemiología. Máster en Salud Ambiental. Dirección \\ Nacional de Salud Ambiental. MINSAP. La Habana, Cuba.
}

\section{RESUMEN}

I ntroducción La nanotecnología llega y son los hombres y mujeres, quienes deben desarrollar y gestionar los avances que traerá.

Objetivos Ofrecer información acerca de las aplicaciones de la nanotecnología para contribuir a la motivación de los profesionales de la salud de pregrado y posgrado en la adquisición y profundización del conocimiento de esta materia.

Fuente de datos Se revisó la literatura y se recopilaron 26 referencias, la mayoría en formato digital y la minoría, de revistas científicas impresa publicadas entre el 2006 y el 2007.

Resultados Se define la nanotecnología como la posibilidad de manejar las cosas a escala molecular, atómica y subatómica. Entre sus campos de aplicación se incluyen: medioambiente, exploración espacial, tecnologías de la comunicación e informática, higiene y salud pública, entre otras. En la actualidad, la comunidad científica internacional la cataloga como uno de los proyectos más innovadores y ambiciosos de la ciencia moderna. La aplicación de la nanotecnología para el diagnóstico, tratamiento, monitoreo y control de sistemas biológicos es denominada nanomedicina, la cual agrupa tres áreas principales: el nanodiagnóstico, la 
liberación controlada de fármacos y la medicina regenerativa. En Cuba, se trabaja en el desarrollo de equipos y metodologías de caracterización de materiales manométricos para la industria biotecnológica.

Conclusiones La nanotecnología puede representar una oportunidad para un país como Cuba, que posee un importante capital humano preparado para enfrentar retos en el campo científico-tecnológico con un enfoque solidario y alto sentido ético.

Palabras clave: Nanotecnología, nanomedicina, nano, nanotubos de carbono.

\section{ABSTRACT}

I ntroduction Nanotechnology is coming and it is the task of men and women to develop and manage the advancements that it will bring.

Objectives To provide information about the nanotechnology application in order to encourage health professionals at undergraduate and graduate levels to acquire and to delve into the knowledge on this subject.

Data source Literature review in which 26 references were collected, mostly in digital format, whereas the rest was taken from printed scientific journals in 2006 and 2007.

Results Nanotechnology is defined as the possibility of handling things at molecular, atomic and subatomic scale. The main fields of application are environment, spatial exploration, communication and informatics technologies, hygiene and public health, among others. At present, the international scientific community considers nanotechnology as one of the most innovative and ambitious projects of the modern science. The application of nanotechnology in diagnosis, treatment, monitoring and control of the biological systems is called nanomedicine, which embraces three main areas, that is, nanodiagnosis, controlled liberalization of drugs, and regenerative medicine. Cuba works for the development of pieces of equipment and methodologies to characterize nanometric materials aimed at the biotechnological industry.

Conclusions Nanotechnology may represent a good opportunity for a country like Cuba with a great deal of skilled human resources capable of facing challenges in the scientific-technological field from a highly ethical and solidarity approach.

Key words: Nanotechnology, nanomedicine, nano, carbon nanotubes.

Sólo tenemos que cerrar los ojos y soñar: el control de la naturaleza a escala atómica promete revolucionar la medicina, mejorar los procesos de depuración y desinfectación del agua, eliminar la polución, aumentar la velocidad de los procesadores y la memoria de los ordenadores...La nanotecnología llega y somos nosotros, hombres y mujeres, quienes debemos desarrollar y gestionar los avances que traerá. ${ }^{1}$

La nanotecnología (deriva del griego nanno que significa enano) es el control de la materia a escalas de entre 1 y 100 nanómetros. $^{2}$ Es la posibilidad de manejar las 
cosas a escala molecular, atómica y subatómica, lo que podría reportar beneficios increíbles a las sociedades presentes y futuras. ${ }^{3}$ Entre sus campos de aplicación se incluyen: medioambiente, exploración espacial, tecnologías de la comunicación e informática, sector energético, textil, construcción y arquitectura, agricultura, ganadería, electrónica, cosmética, industria militar, automovilística, seguridad personal y vial, higiene y salud pública, deportes, espionaje y reducción de la brecha digital, entre otras. ${ }^{4-7,8}$

El término fue acuñado en 1974 por el japonés Taniguchi Norio y fue Richard Feynman (premio Nobel de Física en 1965) con su famosa conferencia titulada "Hay mucho espacio en el fondo", quien marcó un hito para el desarrollo de la nanotecnología, haciendo ver la posibilidad de mover las cosas átomo por átomo. Se considera esta conferencia de Feynman, realizada en 1959, como uno de los referentes teóricos de lo que en la actualidad la comunidad científica internacional cataloga como uno de los proyectos más innovadores y ambiciosos de la ciencia moderna. ${ }^{4-6}$ El gran despertar de la nanotecnología comenzó a partir de los años 80 , a partir del desarrollo de una amplia gama de microscopios de sonda de barrido, que logran imágenes a escala atómica. ${ }^{9}$ El descubrimiento de los nanotubos de carbono (NTC), nanobiosensores de exelentes propiedades mecánicas y eléctricas, ${ }^{7}$ realizado en J apón por Sumio lijima en 1991. Hoy existen cerca de 3 mil productos generados con nanotecnología, la mayoría para usos industriales, aunque las investigaciones más avanzadas se registran en el campo de la medicina y la biología. ${ }^{3}$

En el área de la medicina se han publicado varias investigaciones y resultados de la nanotecnología. Su aplicación en el diagnóstico, tratamiento, monitoreo y control de sistemas biológicos es denominada nanomedicina. ${ }^{10}$ Esta rama de la nanotecnología agrupa tres áreas principales: el nanodiagnóstico, la liberación controlada de fármacos y la medicina regenerativa.

El nanodiagnóstico desarrolla sistemas de análisis y de imagen para detectar una enfermedad o un mal funcionamiento celular en los estadios más tempranos posibles, los nanosistemas de liberación de fármacos transportan los medicamentos sólo a las células o zonas afectadas porque así el tratamiento será más efectivo y con menos efectos secundarios. La medicina regenerativa pretende reparar o reemplazar tejidos y órganos dañados aplicando herramientas nanobiotecnológicas.

Otro gran reto de la nanomedicina es desarrollar nanoherramientas para manipular células, individuales o en grupos de fenotipo común, mediante la interacción específica con los propios nanoobjetos naturales de las células (receptores, partes del citoesqueleto, orgánulos específicos y compartimentos nucleares, entre otros). Ya se están desarrollando nanopinzas y herramientas quirúrgicas de pequeño tamaño que permitirían localizar, destruir o reparar células dañadas. ${ }^{11}$

Para el diagnóstico precoz de enfermedades de una forma selectiva y con un alto nivel de sensibilidad se pueden emplear nanobiosensores como son: puntos cuánticos (CdS), ${ }^{11,12}$ cristales fotónicos, micropalancas, resonadores fotónicos, nanotubos de carbono y nanointerferómetro. En aplicaciones in vivo, las nanopartículas también pueden emplearse para transportar moléculas de metal que se usan como agentes para obtener mejores imágenes del interior del cuerpo humano mediante resonancia magnética, en estos casos, imágenes de tumores de apenas un par de milímetros. Algunos de estos nanoagentes de contraste ya han sido aprobados para su utilización rutinaria en clínica. I gualmente se pueden utilizar para obtener mejores contrates en imágenes óptica, de rayos x y por ultrasonidos. La combinación de estos agentes de imagen con los dispositivos de diagnóstico es 
otra de las líneas emergentes de investigación en nanodiagnóstico. Las nanopartículas se pueden emplear, además, para el diagnostico precoz de la enfermedad de Alzheimer mediante la detección del ligando ADDL, biomarcador específico de dicha enfermedad que aparece en sus primeros estadios. ${ }^{11}$

La investigación en cáncer ilustra muchas de las potencialidades de la nanobiotecnología a largo plazo ya que es de esperar que ayude a desarrollar una terapia anticáncer. En este sentido, se están realizando trabajos de experimentación con láser que han logrado eliminar las células cancerosas respetando las sanas. El trabajo ralizado en la universidad de Stanford, ha utilizado nanotubos de carbono y se ha aprovechado su capacidad para calentarse cuando son expuestos a la luz de un láser. Diferentes investigadores están desarrollando un modelo experimental de linfoma en ratas, para comprobar si mediante la simple exposición de la piel del ratón a la luz láser, son capaces de matar células cancerosas una vez incorporados los nanotubos de carbono. También hablan de la inyección directa en el tumor, por ejemplo en la mama, que sería luego expuesta a la luz cercana al infrarrojo que destruiría el tejido. ${ }^{12-15}$

Baker señaló la reciente creación de lo que él llama "el caballo de Troya de la nanotecnología", en alusión a la capacidad transportadora de un medicamentos que funciona como una nanopartícula, bautizada como dendrímero y que está diseñada para introducir el fármaco anticáncer en las células del tumor, donde su efecto aumenta y la toxicidad disminuye. ${ }^{15}$ I gualmente se han utilizado nanopartículas magnéticas con el recubrimiento adecuado para la localización de las células tumorales. Para ello se recubren las nanopartículas con surfactantes que poseen una zona hidrófila y otra hidrófoba. Una vez que estas nanopartículas se unen a las células cancerosas, se puede inducir su calentamiento mediante un campo magnético de baja intensidad. El calentamiento provoca la destrucción de las células tumorales pero sin causar daño a las células o tejidos sanos circundantes. Estas tecnologías para el tratamiento del cáncer evitarían los graves problemas de efectos secundarios que conllevan los actuales tratamientos de quimioterapia o radioterapia, ${ }^{11,14,15}$ y sus resultados pueden representar un gran logro para el combate contra esta enfermedad. ${ }^{6}$

Entre otros ejemplos de investigaciones en nanomedicina se encuentra el dispositivo desarrollado por Tejai Desai, denominado micropancreas artificial que actúa como biorreactor en miniatura y permite el uso de las células Beta de cualquier dador, con liberación de insulina sin penetración de linfocitos y anticuerpos. ${ }^{16}$

Otra sería la aplicación en la odontología, al existir dentífricos con nanocristales de hidroxiapatita, que es el componente principal de la dentina, presumiblemente, reforzándola. ${ }^{2}$.

En la malaria se está ensayando una vacuna vinculada a nanodispositivos de modo que aumente su estabilidad y prolongue su efecto de inducir inmunogenicidad, además, el tratamiento de la malaria puede ser beneficiado a través de técnicas de encapsulaçion de fármacos con el desarrollo de formas farmacéuticas de liberación controlada por micro y nanosistemas lo que permitiría mejor control de la cinética de liberación del fármaco con menores efectos tóxicos. ${ }^{10} \mathrm{Ha}$ sido propuesta por investigadores, recientemente, la administracion de la vacuna de la hepatitis B a través de una emulsión de gotas microscópicas que se depositan en la nariz. ${ }^{16,17}$

La nanomedicina regenerativa persigue la reparación o reemplazamiento de tejidos y órganos mediante la aplicación de métodos procedentes de terapia génica, terapia 
celular, dosificación de sustancias bio-regenerativas e ingeniería tisular. La terapia génica se basa en utilizar células genéticamente modificadas, la celular en usar células madre y la liberación controlada de sustancias activas, citoquinas y factores de crecimiento propician la reconstrucción tisular. La ingeniera tisular intenta generar tejidos in vivo o in vitro para lo cual necesita materiales biocompatibles que mimetizen respuestas celulares específicas a nivel molecular. Gracias al desarrollo de la nanotecnología los materiales tienen el potencial de interaccionar con componentes celulares, dirigir la proliferación y diferenciación celular y la producción y organización de la matriz extracelular. Entre los materiales que se están utilizando cabe destacar los NTC, nanopartículas como nanohidroxiapatita o nanozirconia, nanofibras de polímeros biodegradables, nanocomposites, entre otros. También se pueden utilizar superficies con nanoestructuración nanométrica que actúen como incubadoras de líneas celulares y favorecen el proceso de diferenciación celular. Los nuevos materiales así obtenidos pueden mejorar la adhesión, duración y tiempo de vida. Algunos ejemplos destacables incluyen polímeros a la nanoescala moldeados en válvulas de corazón y nanocomposites de polímeros para la regeneración ósea. ${ }^{11}$

En cuanto a los riesgos, para Robert Madelin director general de Salud y Protección al Consumidor de la Comisión Europa en Helsinki, la nanotecnología esta aún en pañales. Según este experto, la mayoría de las nanopartículas serán probablemente inocuas, pero hay que estudiar "caso por caso". No descarta "efectos agudos adversos y consecuencias a largo plazo", y subraya que un material puede ser seguro a dimensiones normales y esto no implica que también lo sea su versión nano. ${ }^{2}$ Existe un estudio en el que comprueban experimentalmente en ratones, que los (NTC) mayores a 20 micrones, al no poder ser fagocitados por las células del sistema inmune, se comportan muy similar al asbesto y provocan daños pulmonares y pleurales que incluso podrían desencadenar mesotelioma. Estos resultados tienen una alta probabilidad de ser extrapolables a seres humanos. ${ }^{4}$ Otros autores hacen alusión a mecanismos de neuroproteccion pero a su vez se refieren a la nanoneurotoxicidad como una disciplina emergente, al plantear que recientes observaciones sugieren que las nanoparticulas son capaces de provocar neurotoxicidad y dañar la integridad de la barrera hematoencefálica. Todo lo anterior indica que los efectos de las nanoparticulas no deben ser ignorados ${ }^{18}$ y que la mayoría de las inquietudes se deben a que en la actualidad aún no se conocen profundamente los procesos que rigen la materia en el nivel de la nanoescala a lo cual se le debe hacer frente potenciando la investigación. ${ }^{19}$

Ante su alto grado de complejidad e incertidumbre, la ola de optimismo, estímulo y desarrollo de la nanotecnología a nivel mundial, resulta altamente necesario el estudio, evaluación y debate sobre sus implicaciones sociales, éticas, ambientales y legales. ${ }^{20}$ Es necesario pensar en las regulaciones de la nanotecnología de manera que se puedan cosechar sus beneficios y evitar los daños que pueda causar su mal uso. La propuesta de regulación más conocida la constituyen los "Lineamientos prospectivos sobre la Nanotecnologia molecular". Estos lineamientos contienen supuestos de partida, principios y algunas recomendaciones denominados, por sus autores y promotores, "Desarrollo responsable de la Nanotecnologia molecular". ${ }^{16}$ En cualquier caso, esta incipiente revolución nanotecnológica se debe desarrollar de forma racional y responsable, sin olvidar la relación riesgo-beneficio con la intención de que se aplique para mejorar la calidad de vida de las sociedades actuales y de las generaciones venideras. La bondad o la malignidad de esta tecnología, dependerá de las aplicaciones y fines a los que se destine. ${ }^{3}$

La nanociencia es un área emergente de la ciencia y aunque su impacto en la vida moderna aún parece una historia de ciencia ficción, ${ }^{4}$ el conocimiento de los procesos biológicos, químicos y físicos a nivel molecular, se convertirán en una de 
las revoluciones científicas más importantes para la humanidad, ya se acerca el momento de que sea una realidad palpable y cotidiana, pero para ello, como ciencia multidisciplinaria requiere de las aportaciones de científicos de campos muy diversos. ${ }^{6,18,21,22}$

La Unión Europea en su conjunto se coloca como la primera potencia mundial en el campo de la nanotecnologia ${ }^{23}$ y Estados Unidos, además de Australia, Japón, Corea del Sur, la India, China e Israel, son algunos países que apuestan abierta y estratégicamente por su progreso a través de planes e inversiones destinadas a la investigación y el desarrollo. ${ }^{24}$

En Cuba, en abril de 2006, se celebró la reunión de la red de macrouniversidades públicas de América Latina y el Caribe del área temática de investigación sobre nanotecnología y nuevos materiales, donde asistieron representantes de casi 10 países. ${ }^{7}$. En el país se trabaja además en el desarrollo de equipos y metodologías para la caracterización de materiales nanométricos para la industria biotecnológica. Además, se ha logrado, el encapsulamiento de fertilizantes y factores de crecimiento agrícola, con experimentos realizados a escala de campo en hortalizas; también se creó un método mediante materiales zeolíticos para purificar el agua, el cual fue validado por centros del Ministerio de Salud Pública. En los últimos seis años el Instituto de Ciencia y Tecnología de los Materiales graduó a más de 30 doctores en Ciencias, muchos en el tema de la nanotecnología. ${ }^{25,26}$

La nanotecnología puede representar una oportunidad para un país como Cuba, que posee un importante capital humano preparado para enfrentar retos en el campo científico-tecnológico con un enfoque solidario y alto sentido ético. ${ }^{16}$ De manera general, hoy el tema es una prioridad para un grupo de organismos de la Administración Central del Estado los que a través de sus respectivos representantes integran el Grupo Ad- Hoc sobre Seguridad de las Nanociencias y la Nanotecnología para la Salud, la Alimentación y el Medio Ambiente, figura entre ellos el Ministerio de Salud Pública. Dentro de los objetivos fundamentales de este grupo, están el de realizar el levantamiento de las regulaciones, buenas prácticas, normas de laboratorio, sistemas de protección y otros, a nivel internacional en los campos respectivos a fin de minimizar los impactos negativos, así como identificar experiencias nacionales y regulaciones afines existentes que puedan servir de base al establecimiento de un sistema regulatorio y/o guías para el trabajo seguro en nanotecnología.

Finalmente se puede concluir que la nanotecnología y su aplicación en las ciencias médicas puede resultar de un valor extraordinario en el diagnóstico y tratamiento de enfermedades que constituyen un problema actual en la morbilidad, la mortalidad y la calidad de vida de la población. La investigación en nanotecnología en cuanto a la evaluación de los posibles riesgos a la salud debe ser potenciada. Las regulaciones en materia de nanotecnologia son de gran importancia en los momentos actuales y debe dedicársele especial atención de manera que se puedan cosechar sus beneficios y evitar los daños que pueda causar su mal uso. Cuba dispone del potencial científico y la ética para el desarrollo de la nanotecnología, y sus resultados deben estar encaminados al desarrollo de la ciencia y al bienestar de la sociedad.

\section{REFERENCI AS BI BLI OGRÁFI CAS}


1. Serrano G. Nanotecnología, innovación tecnológica y transformación social en Europa. Nanotecnología y Medio Ambiente. Ética y política de la Nanotecnología [citado J ul 2008]. Disponible en: http://blogs.creamoselfuturo.com/nanotecnologia/2007/11/29/nanotecnologia-y-el-cambio-climatico/

2. Salomone MG. ¿Es segura la nanotecnología? [citado Jul 2008]. Disponible en: http://www.madrimasd.org

3. Cintas Izarra LM. Nanotecnología: la revolución industrial del Siglo XXI. Madrid (UCM), 16 de agosto 2006, No.2,p.11.

4. Marcelo J. Alerta científica sobre los riesgos para la salud y el medio ambiente de la nanotecnologia. Servicio especial [actualizado 2004, citado 8 Ago 2008].

Disponible en: http://www.abc.es

5. Cesare N. Nanotecnologia. Innovación tecnológica y transformación social en Europa [citado Jul 2008]. Disponible en: http://blogs. creamoselfuturo.com/nanotecnologia/2006/10/03/richard-p-feynman-nobel-de-fisica1965/?jal_no_js=true\&poll_id=8

6. Nanotecnología. Nanociencia [citado Jul 2008]. Disponible en: http://www.portalciencia.net

7. Vega Baudrit J. Laboratorio de Nanotecnología (LANOTEC). Costa Rica: Instituto Tecnológico; 2007.

8. Feynman R. Apuntaba a la nanotecnología como la ciencia del futuro. Innovación | átomos. El Mundo. Jueves, 27 de diciembre de 2001, No. 73. Disponible en: http://www.el-mundo.es

9. Alonso I, Avendaño B. Nanotecnología. Correteando entre átomos. 6 de junio de 2006 [citado Jul 2008]. Disponible en:

http://www.bohemia.cubasi.cu/2006/06/06/cienciatecnologia/nanotecnologia. html

10. Figueira Pimentel L, Tavares Jácome A Júnior, Furtado Mosqueira V, SantosMagalhães C, Nereide S. Nanotecnologia farmacéutica aplicada ao tratamento da malária. Rev Brasileira Cienc Farm. 2007;43(4).

11. Lechuga LM, Martínez Alonso C. Nanobiotecnología: Avances Diagnósticos y Terapéuticos. Revista de investigación en Gestión de la Innovación y Tecnología. Nanociencia y Nanotecnologia II. 2006(35). Marzo-Abri I.

12. Zhang $H$, Douglas $Y$, Wang $C$. Quantum dots for cancer diagnosis and therapy: biological and clinical perspectives. Nanomedicine serie en Internet . 2008 citado 2008 ; 3(1):83-91. Disponible en: http://www.ncbi.nlm.nih.gov/pubmed/18393668

13. Sumer, B, Gao J. Theranostic nanomedicine for cancer. Nanomedicine serie en Internet . 20020082008 citado 2008 ;3(2): 137-40. Disponible en:

http://www.ncbi.nlm.nih.gov/pubmed/18373419?dopt=Abstract

14. Marco J. Cáncer terapias experimentales. La nanotecnología se incorpora al tratamiento del cáncer [citado Jul 2008]. Disponible en: http://www.elmundo.es 
15. Permite nanotecnología luchar contra cáncer [citado J ul 2008]. Disponible en: http://www.eluniversal.com/index.html

16. Ministerio de Ciencia, Tecnología y Medio Ambiente (CITMA). Elementos iniciales para el análisis sobre la Nanotecnologia en Cuba. La Habana: CITMA; 2002. Disponible en: http://www.occyt.cu

17. Gotas microscópicas ofrecen resultados prometedores como vacuna contra hepatitis B. Washington, agosto 13/2008 (EFE). Disponible en:

http://www.sld.cu/servicios/aldia/view.php?idn=22695

18. Hari Shanker Sharma . Nanoneuroscience: emerging concepts on nanoneurotoxicity and nanoneuroprotection. Nanomedicine serie en Internet . 20020082007 citado 2008 ;2(6):753-8. Disponible en: http://www. futuremedicine.com/toc/nnm/2/6

19. Fernández Agis D, Fernández Castillo A. La Nanotecnologia, inquietudes sociales y problemas éticos derivados. El Catoblepas. Marzo 2007, No.61, p.14. Disponible en: http://www.nodulo.org/ec/2007/n061.htm

20. Delgado Ramos GC. Nanotecnología, paradigma tecnológico de vanguardia. Contribuciones a la Economía [citado Jul 2008]. Disponible en:

http://www.eumed.net/ce/2007a/gcdr.htm

21. De las Fuentes A. El año de la Nanotecnologia. El suplemento para comprender el mundo digital [actualizado 2001, citado Jul 2008]. Disponible en: http://www.elmundo.es

22. Nanotecnologia. De Wikipedia, la enciclopedia libre [citado Jul 2008]. Disponible en: http://www.wikipedia.org

23. Alonso, Andaluz DJ, Da Sánchez Páramo J. Nanotecnología en España. Revista de investigación en Gestión de la Innovación y Tecnología. Nanociencia y Nanotecnologia I, enero-febrero de 2006, No.34.

24. Pedreño A. Repercusiones económicas de los avances en Nanotecnología [citado Jul 2008]. Disponible en: http://www.iei.ua.es

25. Incursiona Cuba en la Nanotecnologia. Granma Internacional Versión Digital. 17 de Marzo de 2008[ citado Ago 2008]. Disponible en: http://www.granma.cu/espanol/2008/marzo/lun17/nanotecnologia-e.html

26. Pérez Sáez D. La era del nano [citado Jul 2008]. Disponible en: http://www.juventudrebelde.cu/

Recibido: 15 de septiembre de 2008 .

Aprobado: 26 de septiembre de 2009. 
Yoerquis Mejias Sánchez. Dirección Nacional de Ciencia y Técnica. MINSAP. La Habana, Cuba.

E-mail: amtoledo@infomed.sld.cu 